\title{
FUNCTIONS THAT PRESERVE THE UNIFORM DISTRIBUTION OF SEQUENCES
}

\author{
WILLIAM BOSCH
}

\begin{abstract}
In this paper, necessary and sufficient conditions are given for certain functions to preserve the uniform distribution of sequences. An analytic condition allows the construction of examples. An application is also given.
\end{abstract}

1. Introduction. If $\left(x_{n}\right)$ is a sequence of points contained in the interval $I=$ $[0,1)$ and $f: I \rightarrow I$ is a function, then $\left(f\left(x_{n}\right)\right)$ is also a sequence in $I$. Generally, one can investigate properties which $\left(f\left(x_{n}\right)\right)$ inherits from $\left(x_{n}\right)$ under certain conditions on the function $f$.

In this paper, we consider sequences which are uniformly distributed in $I$ and ask what conditions on $f$ are necessary or sufficient to imply $\left(f\left(x_{n}\right)\right)$ is also uniformly distributed in $I$. Such functions may be useful in constructing uniformly distributed sequences or in checking a sequence for uniform distribution. An application is given in this paper.

We use the terminology and notation of Kuipers and Niederreiter [1] adapted to suit our purposes.

First we restate the definition of uniformly distributed sequences $[\mathbf{1}, \mathrm{p} .1]$.

DEFINITION 1.1. A sequence $w=\left(x_{n}\right), n=1,2, \ldots$, of real numbers contained in $[0,1)$ is said to be uniformly distributed in $[0,1)$ if for every pair $a, b$ of real numbers with $0 \leq a<b \leq 1$, we have

$$
\lim _{N \rightarrow+\infty} \frac{A([a, b) ; N ; w)}{N}=b-a .
$$

In this definition, $A([a, b) ; N ; w)$ denotes the number of terms $x_{n}, 1 \leq n \leq N$, that belong to $[a, b)$.

The condition in Definition 1.1 is equivalent to the analogous condition using either open $(a, b)$ or closed $[a, b]$ subintervals of $[0,1)[\mathbf{1}, \mathbf{p} .6]$. In later arguments we will find it convenient to use open subintervals.

Throughout we use $I_{k}, k=1,2, \ldots$, to denote open, half-open, or closed subintervals of $[0,1], I$ to denote $[0,1)$, and $C(I)$ to denote $[0,1]$.

Definition 1.1 can also be formulated for sequences in $C(I)$. Then any sequence uniformly distributed in $I$ is still uniformly distributed in $C(I)$. The converse is also true no matter how the terms of the sequence which equal one are handled. Those terms can be equated to a value in $I$ (such as zero) or simply deleted. Consequently, we can be relaxed about the specific details of Definition 1.1.

Received by the editors February 13, 1987.

1980 Mathematics Subject Classification (1985 Revision). Primary 11K06; Secondary 11J71.

Key words and phrases. Uniform distribution, functions, preserve.

ACKNOWLEDGEMENT. The author thanks the referee for substantive help in correcting the original version of this manuscript. 
We will make use of the following criterion for the uniform distribution of sequences in $I[\mathbf{1}$, p. 3]. if

THEOREM 1.2. The sequence $\left(x_{n}\right) \subseteq I$ is uniformly distributed in $I$ if and only

$$
\lim _{N \rightarrow+\infty} \frac{1}{N} \sum_{n=1}^{N} f\left(x_{n}\right)=\int_{0}^{1} f(x) d x
$$

holds for all functions $f$ Riemann-integrable on $C(I)$.

The next definition and lemma give a generalized version of the condition in Definition 1.1 .

DEFINITION 1.3. The sequence $\left(a_{i}\right) \subseteq C(I), i=1,2, \ldots$, is a partition of $C(I)$ if it satisfies one of the following conditions:

(a) $\left(a_{i}\right)$ is a finite sequence;

(b) $\left(a_{i}\right)$ is an infinite, strictly monotone increasing sequence tending to one;

(c) $\left(a_{i}\right)$ is an infinite, strictly monotone decreasing sequence tending to zero;

(d) $\left(a_{i}\right)$ is a bi-infinite sequence indexed by the set of integers such that $a_{i}<a_{i+1}$ for all integers $i,\left(a_{i}\right)$ tends to one as $i$ tends to infinity, and $\left(a_{i}\right)$ tends to zero as $i$ tends to negative infinity.

In case (a) of Definition 1.3, it will be convenient to adjoin the terms zero and one to the sequence $\left(a_{i}\right)$ as the first and last terms. In case (b), we adjoin zero as the first term $a_{0}$. In case (c), we adjoin one as $a_{0}$. We assume this has been done.

LEMMA 1.4. Let $\left(a_{i}\right)$ be a partition of $C(I)$ (in the sense of Definition 1.3). Let $w=\left(x_{n}\right)$ be a sequence in $I$. The sequence $w$ is uniformly distributed in $I$ if and only if

$$
\lim _{N \rightarrow+\infty} \frac{A((b, c) ; N ; w)}{N}=c-b
$$

for all $b, c$ such that $a_{i} \leq b<c \leq a_{i+1}$ for some $i$; and

$$
\lim _{N \rightarrow+\infty} \frac{A(F ; N ; w)}{N}=0
$$

for any finite subset $F$ of the union $\bigcup\left\{a_{i}\right\}$.

PROOF. If $w$ is a uniformly distributed sequence in $I$, (a) follows immediately from Definition 1.1. To verify (b), we note the characteristic function of the set of terms $\bigcup\left\{a_{i}\right\}$ of the sequence $\left(a_{i}\right)$ is Riemann-integrable over $C(I)$. Using Theorem 1.2 , we obtain the limit in (b) for the set $\bigcup\left\{a_{i}\right\}$ and hence for any subset $F$ of $\bigcup\left\{a_{i}\right\}$.

For the converse, note that any subinterval of $I$ of the form $(a, b)$, with $0<a<$ $b<1$, can be written as a finite union of disjoint subintervals (with total length equal to $b-a)$ of the type in (a) and a finite subset of $\bigcup\left\{a_{i}\right\}$. Conditions (a) and (b) then imply the limit in Definition 1.1 holds for those subintervals. Then one can show that the limit in Definition 1.1 holds for subintervals of $I$ of the form $[a, b)$ with $0<a<b<1$. This is enough to ensure that the sequence $w$ is uniformly distributed in $I[\mathbf{1}$, p. 6].

We now give a result on the Riemann-integrability of the characteristic function of certain sets. 
If $\left\{I_{k}\right\}$ is a sequence of disjoint subintervals of $I$ and the sequence of left endpoints of the $I_{k}$ form a partition of $C(I)$, then so do the right endpoints. For convenience, we will call such a sequence a monotone sequence of disjoint intervals.

THEOREM 1.5. If $\left\{I_{k}\right\}$ is a monotone sequence of disjoint subintervals of $I$, then the characteristic function of their union is Riemann-integrable over $C(I)$.

PROOF. The characteristic function has at most a countable number of discontinuities and is therefore Riemann-integrable on $[0,1]$.

2. The main results. In this section, we derive necessary and sufficient conditions for a function to have the property that if $\left(x_{n}\right)$ is uniformly distributed in $I$, then so is $\left(f\left(x_{n}\right)\right)$. Such a function will be said to preserve the uniform distribution of sequences.

We will restrict much of our discussion to the following class of functions:

DEFinition 2.1. A function $f: I \rightarrow C(I)$ belongs to the class $G$ if and only if there is a partition $B_{f}=\left(b_{j}\right)$ of $C(I)$ corresponding to $f$ such that

(a) $(c, d) \subseteq\left(b_{j}, b_{j+1}\right)$, for any $j$ implies $f^{-1}((c, d))=\bigcup I_{k}, k=1,2, \ldots$, where $\left\{I_{k}\right\}$ is a monotone sequence of disjoint intervals in $C(I)$; and

(b) for each $j, f^{-1}\left(b_{j}\right)$ is empty or is a partition of $C(I)$.

REMARKS. Conditions (a) and (b) of Definition 2.1 are not stringent. For example, a continuous function $f: I \rightarrow C(I)$ belongs to $G$ provided $f^{-1}(b)$ is finite for all $b$ in $C(I)$. In fact, the motivation for part (a) of Definition 2.1 is that continuous functions have this property automatically $\left(I_{k}\right.$ can be an open, halfopen, or closed interval). Discontinuous functions such as $f(x)=n(n+1) x-\left(n^{2}-1\right)$, $(n-1) / n \leq x<n /(n+1), n \geq 1$, also belong to $G$. The function

$$
f(x)= \begin{cases}x, & x \neq 1 / 2 \\ 1 / 4, & x=1 / 2\end{cases}
$$

belongs to $G$ with $B_{f}=\left\{0, \frac{1}{4}, 1\right\}$ as a corresponding partition and suggests the motivation for partitioning the range of the function.

We could enlarge the class $G$ by replacing the monotone condition on the sequence $\left\{I_{k}\right\}$ in (a) by the condition that the characteristic function of $\bigcup I_{k}$ be Riemann-integrable on $[0,1]$. We prefer the monotone condition because it is used in our main results and parallels the partitions defined in Definition 1.3.

Not all functions in $G$ preserve the uniform distribution of sequences. In the next definition and theorem, we isolate the key property of the functions in $G$ that do preserve the uniform distribution of sequences.

DEFINITION 2.2. Let $f$ be in $G$ with $B_{f}=\left(b_{j}\right)$ as a corresponding partition of $C(I)$. Suppose for all $(c, d) \subseteq\left(b_{j}, b_{j+1}\right)$ and $f^{-1}((c, d))=\bigcup I_{k}$, we have

$$
d-c=\sum m\left(I_{k}\right)
$$

where $m\left(I_{k}\right)$ denotes the measure (length) of the interval $I_{k}$ and the summation extends over appropriate values of $k$. Then we say the measure (or length) of intervals is preserved under inverse images of $f$.

Note that the convergence of the series is assured. 
THEOREM 2.3. A function $f$ in $G$ preserves the uniform distribution of sequences if and only if the measure of intervals is preserved under inverse images of $f$.

PROOF. Let $f$ be in $G$ and suppose $B_{f}=\left(b_{j}\right)$ is a corresponding partition of $C(I)$ for which (a) and (b) of Definition 2.1 hold. Let $(c, d)$ be an arbitrary open subinterval of one of the $\left(b_{j}, b_{j+1}\right)$ and let $S=\bigcup I_{k}=f^{-1}((c, d))$. By Theorem 1.5 , the characteristic function $X_{S}$ of $S$ is Riemann-integrable over $C(I)$.

Let $w=\left(x_{n}\right)$ be a uniformly distributed sequence in $I$ and let $w^{*}=\left(f\left(x_{n}\right)\right)$. Using Theorem 1.2, we calculate as follows:

$$
\begin{aligned}
m(S) & =\int_{0}^{1} X_{S} d x=\lim _{N \rightarrow+\infty} \frac{1}{N} \sum_{n=1}^{N} X_{S}\left(x_{n}\right) \\
& =\lim _{N \rightarrow+\infty} \frac{A(S ; N ; w)}{N}=\lim _{N \rightarrow+\infty} \frac{A\left((c, d) ; N ; w^{*}\right)}{N} .
\end{aligned}
$$

Now suppose $f$ preserves the uniform distribution of sequences. Then the last limit in the preceding calculation equals $d-c$. Hence the measure of intervals is preserved under inverse images of $f$.

Conversely, if the measure of intervals is preserved under inverse images of $f$, then

$$
d-c=\sum m\left(I_{k}\right)=\lim _{N \rightarrow+\infty} \frac{A\left((c, d) ; N ; w^{*}\right)}{N} .
$$

Hence the sequence $w^{*}$ satisfies (a) of Lemma 1.4 relative to the partition $B_{f}$.

Now consider $f^{-1}\left(b_{j}\right)$ for a fixed $b_{j}$ in $B_{f}$. The set $f^{-1}\left(b_{j}\right)$ is empty or is a partition of $C(I)$. If the latter is the case, the proof of Lemma 1.4 implies

$$
\lim _{N \rightarrow+\infty} \frac{A\left(f^{-1}\left(b_{j}\right) ; N ; w\right)}{N}=0 .
$$

This is also obviously true if $f^{-1}\left(b_{j}\right)$ is empty. But $f\left(x_{n}\right)=b_{j}$ if and only if $x_{n}$ belongs to $f^{-1}\left(b_{j}\right)$. So

$$
\lim _{N \rightarrow+\infty} \frac{A\left(\left\{b_{j}\right\} ; N ; w^{*}\right)}{N}=0 .
$$

Hence, for any finite subset $F$ of $B_{f}$,

$$
\lim _{N \rightarrow+\infty} \frac{A\left(F ; N ; w^{*}\right)}{N}=0 .
$$

Thus $w^{*}$ also satisfies (b) of Lemma 1.4. Hence $w^{*}$ is uniformly distributed in $I$ and $f$ preserves the uniform distribution of sequences.

EXAMPLE 2.4. Theorem 2.3 implies the function $f(x)=n(n+1) x-\left(n^{2}-1\right)$, $(n-1) / n \leq x<n /(n+1), n \geq 1$, preserves the uniform distribution of sequences.

COROLlaRY 2.5. Let $f: I \rightarrow I$ be continuous and one-to-one. The function $f$ preserves the uniform distribution of sequences if and only if $f(x) \equiv x$ or $f(x) \equiv$ $1-x$ on $I$.

PROOF. The function $f$ belongs to $G$ and maps intervals onto intervals in a oneto-one fashion. If $f$ preserves the uniform distribution of sequences, Theorem 2.3 shows that $f$ itself preserves the length of intervals. Because of that fact, $f(x) \equiv x$ or $f(x) \equiv 1-x$ on $I$. The converse is clear. 
Corollary 2.5 shows that no function of the form $f(x)=x^{p}, p>0, p \neq 1$ preserves the uniform distribution of sequences. Thus if $\left(x_{n}\right) \subseteq I$ is uniformly distributed, then $\left(x_{n}^{p}\right), p>0, p \neq 1$ is not uniformly distributed.

Theorem 2.3 (and its consequences) relate the preservation of the uniform distribution of sequences by functions of $G$ to the preservation of the measure of intervals under inverse images of the functions. The object of the next several results is to relate the preservation of the measure of intervals under inverse images of a function to analytic properties of the function. We begin with theorems about functions which may or may not belong to the class $G$.

THEOREM 2.6. Let $f: I \rightarrow C(I)$ preserve the uniform distribution of sequences. Suppose for some subinterval $J$ of $C(I), f^{-1}(J)=S \cup T$, where $S$ is a subinterval of $I$ and $T$ is any subset of $I$ with $S \cap T=\varnothing$. Then $m(S) \leq m(J)$.

PROOF. If $w=\left(x_{n}\right)$ is a uniformly distributed sequence in $I$, then so is $w^{*}=$ $\left(f\left(x_{n}\right)\right)$. Since $A(S ; N ; w) \leq A\left(J ; N ; w^{*}\right)$, we can divide both sides of this inequality by $N$, let $N$ tend to infinity, and obtain the result.

THEOREM 2.7. Let $f: I \rightarrow C(I)$ preserve the uniform distribution of sequences. Suppose $0 \leq a<1, f^{\prime}(a)$ exists, and $f$ is continuous on an interval containing $a$. Then $\left|f^{\prime}(a)\right| \geq 1$.

PROOF. By using the definition of the derivative, one can show that if $\left|f^{\prime}(a)\right|<1$, then $f$ maps an interval onto an interval of lesser length. This contradicts Theorem 2.6 .

Theorem 2.7, the Intermediate Value Theorem for Derivatives, and Corollary 2.5 imply the following:

COROLlaRY 2.8. Let $f: I \rightarrow C(I)$ be continuous on $I$ and differentiable in $(0,1)$. If $f$ preserves the uniform distribution of sequences in $I$, then $f(x) \equiv x$ or $f(x) \equiv 1-x$ on $I$.

Theorem 2.7, Corollary 2.8, and Example 2.4 suggest a look at piecewise differentiable functions with derivatives having absolute value bounded below by one. This leads to one of our main results.

THEOREM 2.9. Let $f: I \rightarrow C(I)$ and suppose $A=\left\{a_{0}, a_{1}, \ldots, a_{m}\right\}$ is a finite partition of $C(I)$ such that $f$ is continuously differentiable and $\left|f^{\prime}(x)\right| \geq 1$ on each $\left(a_{i}, a_{i+1}\right), i=0,1, \ldots, m-1$. The function $f$ preserves the uniform distribution of sequences in $I$ if and only if there is a finite partition $B$ of $C(I)$ such that $y$ in $C(I) \backslash B$ implies $f^{-1}(y)$ is not empty, $f^{\prime}$ exists at each point of $f^{-1}(y)$, and

$$
1=\sum \frac{1}{\left|f^{\prime}(x)\right|}
$$

where the summation extends over all preimages $x$ of $y$.

PROOF. We begin with some general remarks and notation. Since $\left|f^{\prime}(x)\right| \geq 1$ on each $\left(a_{i}, a_{i+1}\right), f$ restricted to such an interval is one-to-one and continuously differentiable. The function $f$ maps the interval $\left(a_{i}, a_{i+1}\right)$ onto some open subinterval $\left(c_{i}, d_{i}\right)$ of $I$. Now $f$ restricted to $\left(a_{i}, a_{i+1}\right)$ has an inverse $g_{i}$ defined on $\left(c_{i}, d_{i}\right)$. The function $g_{i}$ is continuously differentiable on $\left(c_{i}, d_{i}\right)$ and, since its derivative is 
bounded, is uniformly continuous on $\left(c_{i}, d_{i}\right)$. Thus $g_{i}$ can be extended to a function continuous on $\left[c_{i}, d_{i}\right]$. We shall assume this extension has been made.

If $y$ is in $\left(c_{i}, d_{i}\right)$, then $g_{i}^{\prime}(y)=1 / f^{\prime}(x)$, where $x$ is in $\left(a_{i}, a_{i+1}\right)$ and $f(x)=y$.

Let $C$ denote the set of endpoints of the intervals $\left[c_{i}, d_{i}\right]$ and let $D=\left\{f\left(a_{i}\right): i=\right.$ $0,1, \ldots, m\}$. Denote $C \cup D$ by $B=\left\{b_{k}\right\}$ for appropriate indices $k$. We can assume $b_{k}<b_{k+1}$ for all indices $k$ except the last. For each $k$ there is a subset $\left\{h_{j k}\right\}$ of $\left\{g_{i}\right\}$ such that if $(b, c)$ is any subinterval of $\left(b_{k}, b_{k+1}\right)$, we can write

$$
m\left(f^{-1}((b, c))\right)=\sum_{j}\left|h_{j k}(c)-h_{j k}(b)\right| .
$$

Since each $g_{i}$ is monotone, so is each $h_{j k}$ and we can write the sum in (2.1) as a sum of increasing functions less a sum of decreasing functions. Then we can apply the Mean Value Theorem for Derivatives to obtain a $u$ in $(b, c)$ such that

$$
m\left(f^{-1}((b, c))\right)=(c-b) \sum_{j}\left|h_{j k}^{\prime}(u)\right| .
$$

We are now ready to prove the theorem. First, suppose $f$ preserves the uniform distribution of sequences in $I$. Then if $\left(x_{n}\right)$ is a uniformly distributed sequence in $I$, every subinterval $(a, b)$ of $(0,1)$ contains members of $\left(f\left(x_{n}\right)\right)$. From this we deduce that $B$ is a partition of $C(I)$ containing $\{0,1\}$. Clearly $f$ belongs to $G$ with $B$ as a corresponding partition. We now verify that $B$ has the properties asserted.

Let $y \in C(I) \backslash B$. If $f^{-1}(y)$ is empty, then $y \notin C(f(I))$. Thus there is an open interval $J$ in $I$ such that $J \cap f(I)=\varnothing$. But this contradicts the assumption that $f$ preserves the uniform distribution of sequences. Thus $f^{-1}(y)$ is not empty.

It is also evident that $f^{-1}(y)$ is a finite subset of the union of the intervals $\left(a_{i}, a_{i+1}\right), i=0,1, \ldots, m-1$. Thus $f^{\prime}$ exists at each point of $f^{-1}(y)$.

Finally, there is an interval of the form $[y, c]$ contained in $C(I) \backslash B$. Then, using Theorem 2.3 and equality (2.2), we have

$$
1=\sum_{j}\left|h_{j k}^{\prime}(u)\right|
$$

for some $u$ in $(y, c)$. Letting $c$ tend to $y$, we get

$$
1=\sum \frac{1}{\left|f^{\prime}(x)\right|}
$$

where the summation extends over the preimages $x$ of $y$.

Conversely, let us assume there is a finite partition $B^{*}$ of $C(I)$ such that $y$ in $C(I) \backslash B^{*}$ implies $f^{-1}(y)$ is not empty, $f^{\prime}(x)$ exists at each point of $f^{-1}(y)$, and such that (2.3) holds for each $y$ in $C(I) \backslash B^{*}$. Let $B$ denote the union of $B^{*}, C$, and $D$. The function $f$ belongs to $G$ with $B$ as the corresponding partition. In order to apply Theorem 2.3 , we need only show that if $(b, c)$ is any subinterval of $C(I) \backslash B$, then the measure of $(b, c)$ is preserved under inverse images of $f$. This is accomplished by using (2.2).

REMARK. Theorems 2.7 and 2.9 can be formulated in terms of one-sided derivatives. The statements and proofs are essentially the same as those given.

EXAMPLE 2.10. Using the differential equation in Theorem 2.9, we can construct functions which are not piecewise linear and which preserve the uniform distribution 
of sequences. One such example is

$$
f(x)= \begin{cases}\frac{4 x(x+1)}{3}, & 0 \leq x \leq \frac{1}{2}, \\ \frac{(7-8 x)+\sqrt{49-48 x}}{8}, & \frac{1}{2}<x \leq 1 .\end{cases}
$$

Since the length of intervals is preserved under inverse images of this function, $L_{1}+L_{2} \equiv L_{3}$ (see Figure 1).

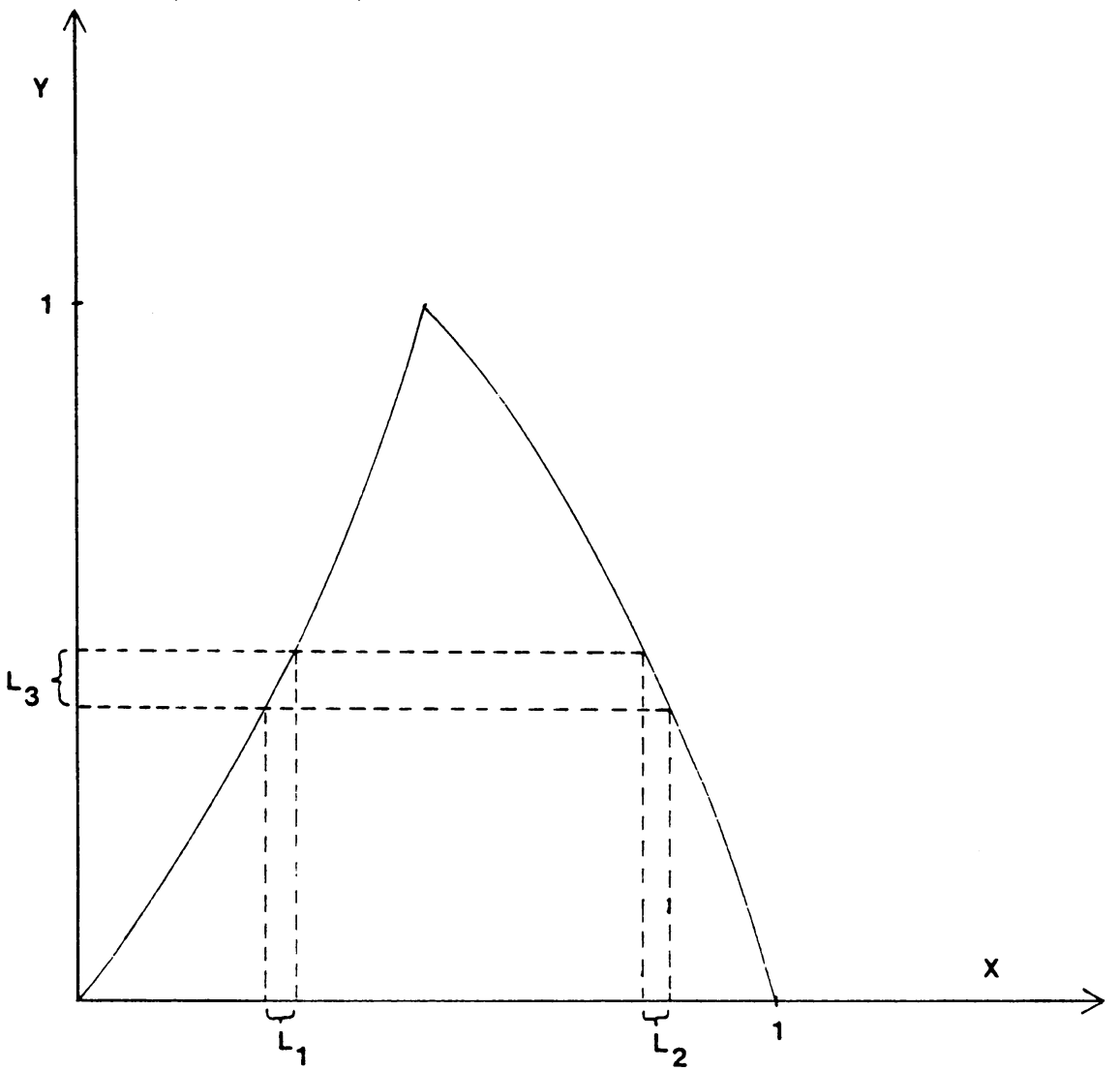

FIGURE 1

3. Additional results and an application. In $\S 2$, we considered functions which are continuously differentiable at all but a finite number of points of $I$. Thus the sum in (2.1) and associated sums are finite. This facilitates the derivation of the differential equation in Theorem 2.9. If those sums are infinite, we face interchanges of limiting processes.

In this section, we consider a class of functions continuously differentiable on $I$ except for a set of points forming a partition of $C(I)$ in the sense of $(\mathrm{d})$ of Definition 1.3. The cases (b) and (c) of Definition 1.3 lead to similar results.

We use the same notation as in Theorem 2.9. In particular, the sets $C, D$, and $B$ have the same meaning as in Theorem 2.9. However, $B$ may be infinite and is not automatically a partition of $C(I)$. We make the further assumption that $B$ is a partition of $C(I)$. 
With these assumptions, we can state and prove this result:

THEOREM 3.1. Let $f: I \rightarrow C(I)$ and suppose $\left(a_{i}\right)$ is a bi-infinite partition of $C(I)$ such that $f$ is continuously differentiable and $\left|f^{\prime}(x)\right| \geq 1$ on each $\left(a_{i}, a_{i+1}\right)$. For each $y \in C(I) \backslash B$, define

$$
h(y)=\sum \frac{1}{\left|f^{\prime}(x)\right|}
$$

where the summation extends over all preimages $x$ of $y$. If $f$ preserves the uniform distribution of sequences, then

(a) $h(y) \leq 1$ for all $y$ in $C(I) \backslash B$, and

(b) there is a dense subset $S$ of $C(I) \backslash B$ such that $h$ is continuous and identically 1 on $S$.

ProOF. Evidently $f$ belongs to $G$ with $B$ as a corresponding partition.

(a) If $h(y)>1$ for some $y$ in $C(I) \backslash B$, then $y$ is in $\left(b_{k}, b_{k+1}\right)$ for some $k$ and there is a $N$ such that

$$
1<\sum_{j=1}^{N}\left|h_{j k}^{\prime}(y)\right| .
$$

Since each $h_{j k}^{\prime}$ is continuous, we can find a subinterval $(b, c)$ containing $y$ and contained in $\left(b_{k}, b_{k+1}\right)$ such that

$$
1<\sum_{j=1}^{N}\left|h_{j k}^{\prime}(u)\right|
$$

on $(b, c)$. Using $(2.2)$, we find $m\left(f^{-1}(b, c)\right)>(c-b)$. This contradicts Theorem 2.3 and proves part (a).

(b) The function $h(y)$ is defined by a convergent series of continuous functions. A standard result (see e.g. [2, p. 141]) indicates that there is a dense subset of each subinterval of $C(I) \backslash B$ on which $h(y)$ is continuous. The union of these subsets is a dense subset $S$ of $C(I)$.

If $h(y)<1$ for some $s \in S$, then $h(y)<1$ on some subinterval of $C(I) \backslash B$. Consequently, there is a subinterval $(b, c) \subseteq\left(b_{k}, b_{k+1}\right)$ of $C(I) \backslash B$ and a constant $r$ such that

$$
\sum_{j}\left|h_{j k}^{\prime}(y)\right| \leq r<1
$$

on $(b, c)$. By using partial sums of the series (2.1) and the corresponding form of (2.2), we deduce

$$
m\left(f^{-1}(b, c)\right) \leq r(c-b)<(c-b) .
$$

This also contradicts Theorem 2.3 and proves part (b).

The next example shows that the convergence of the series in Theorem 3.1 need not be uniform on each $\left(b_{k}, b_{k+1}\right)$ and $h(y)<1$ is indeed possible for some $y$ in $C(I) \backslash B$.

EXAMPLE 3.2. For each $n \geq 3$, let $h_{n}$ denote the function defined on $C(I)$ with graph consisting of the four straight line segments joining the points $(0,1)$, 
$(1 / 2-1 / n, 1-1 / n),(1 / 2,1 / 2-1 / n),(1 / 2+1 / n, 1-1 / n)$, and $(1,1)$ in succession. Also define $h_{2} \equiv 0$. Next let

$$
\begin{gathered}
g_{n}^{*}(x)=\int_{0}^{x}\left[h_{n}-h_{n-1}\right] \\
g_{n}(x)=g_{n}^{*}(x)+\left(1-\sum_{k=3}^{n} g_{k}^{*}(1)\right),
\end{gathered}
$$

for $n \geq 3$. If $g_{n}(1)$ is denoted by $a_{n}$, it follows that $g_{n}:[0,1] \rightarrow\left[a_{n+1}, a_{n}\right]$ and that $\left(a_{n}\right)$ is an infinite partition of $C(I)$ in the sense of $(c)$ of Definition 1.3. We also have $0<g_{n}^{\prime}(x)<1$ on $(0,1)$. Define $f$ on $\left(a_{n+1}, a_{n}\right]$ by $f=g_{n}^{-1}$ for $n \geq 3$. Then $f$ belongs to $G$ with $B=\{0,1\}$ as the corresponding partition of $C(I)$. It can also be shown directly that the measure of intervals in $(0,1)$ is preserved under inverse images of $f$. Thus $f$ preserves the uniform distribution of sequences.

The function $h(y)$ defined in Theorem 3.1 is, for this case, a series with $n$th partial sum equal to $h_{n}, n \geq 3$. This series converges to 1 on $\left(0, \frac{1}{2}\right) \cup\left(\frac{1}{2}, 1\right)$, but not uniformly. Also, $\frac{1}{2}$ is in $C(I) \backslash B$ but $h\left(\frac{1}{2}\right)=\frac{1}{2} \neq 1$. So $h$ is discontinuous at $y=\frac{1}{2}$.

As an application of Theorem 3.1, we offer the following solution to Problem 7.20 in $[\mathbf{1}$, p. 68].

A PPLICATION 3.3. Let $\left(x_{n}\right)$ be a uniformly distributed sequence in $(0,1)$. The fractional parts of the reciprocals $\left(\left\{1 / x_{n}\right\}\right)$ is not a uniformly distributed sequence in $I$.

Solution. Define $f:(0,1] \rightarrow[0,1)$ by $f(x)=(1-n x) / x$, for $1 /(n+1)<x \leq$ $1 / n$ and $n \geq 1$. Note that $f(x)=\{1 / x\}$ and so $\left(f\left(x_{n}\right)\right)=\left(\left\{1 / x_{n}\right\}\right)$. The function $f$ is of the type covered by a variant of Theorem 3.1 .

If $y$ is in $(0,1)$,

$$
f^{-1}(y)=\left\{\frac{1}{y+n}: n \geq 1\right\}
$$

Thus, if $h(y)$ is defined as in Theorem 3.1,

$$
h(y)=\sum_{n=1}^{+\infty} \frac{1}{(y+n)^{2}}
$$

But this latter series is a decreasing and continuous function of $y$ and cannot satisfy the conclusion of Theorem 3.1. Hence $f$ does not preserve the uniform distribution of sequences and $\left(\left\{1 / x_{n}\right\}\right)$ is not uniformly distributed in $I$.

We offer the next theorem as a converse of Theorem 3.1. Here $B$ denotes the same set as in Theorem 3.1 and is again assumed to be a partition of $C(I)$.

THEOREM 3.4. Let $f: I \rightarrow C(I)$ and suppose $\left(a_{i}\right)$ is a bi-infinite partition of $C(I)$ such that $f$ is continuously differentiable and $\left|f^{\prime}(x)\right| \geq 1$ on each $\left(a_{i}, a_{i+1}\right)$. For each $y$ in $C(I) \backslash B$, define

$$
h(y)=\sum \frac{1}{\left|f^{\prime}(x)\right|}
$$

where the summation extends over all preimages $x$ of $y$. If $h(y) \equiv 1$ and the convergence is uniform on each subinterval of $C(I) \backslash B$, then $f$ preserves the uniform distribution of sequences. 
ProOF. Let $(b, c) \subseteq\left(b_{k}, b_{k+1}\right) \subseteq C(I) \backslash B$. Given $\varepsilon>0$, choose $N$ so large that $n>N$ implies that

$$
m\left(f^{-1}(b, c)\right)-\varepsilon \leq \sum_{j=1}^{n}\left|h_{j k}(c)-h_{j k}(b)\right| \leq m\left(f^{-1}(b, c)\right) .
$$

Then

$$
m\left(f^{-1}(b, c)\right)-\varepsilon \leq \sum_{j=1}^{n}\left|h_{j k}^{\prime}\left(u_{n}\right)\right|(c-b) \leq m\left(f^{-1}(b, c)\right) .
$$

Letting $n$ tend to infinity, we obtain

$$
m\left(f^{-1}(b, c)\right)-\varepsilon \leq(c-b) \leq m\left(f^{-1}(b, c)\right) .
$$

Since $\varepsilon$ was arbitrary, $m\left(f^{-1}(b, c)\right)=(c-b)$. Since $f$ belongs to $G$, this proves that $f$ preserves the uniform distribution of sequences.

\section{REFERENCES}

1. L. Kuipers and H. Niederreiter, Uniform distribution of sequences, Wiley, New York, 1974.

2. H. L. Royden, Real analysis, 2nd ed., Macmillan, New York, 1968.

Department of Mathematics and Applied Statistics, University of NorthERN Colorado, Greeley, Colorado 80639 